\title{
MULTIDISCIPLINARY CONTROL OF A SPARSE INTERFEROMETRIC ARRAY SATELLITE TESTBED
}

\author{
Soon-Jo Chung ${ }^{*}$, David Lobosco ${ }^{\dagger}$ and David W. Miller ${ }^{t}$ \\ Space Systems Laboratory, Department of Aeronautics and Astronautics, MIT \\ 77 Mass Avenue, Cambridge, Massachusetts 02139 \\ Carl Blaurock ${ }^{\S}$ \\ Midé Technology Corporation, 200 Boston Avenue, Medford, Massachusetts 02155
}

\begin{abstract}
The MIT Adaptive Reconnaissance Golay-3 Optical Satellite (ARGOS) is a wide-angle Fizeau interferometer spacecraft testbed. Designing a space-based interferometer, which requires such high tolerances on pointing and alignment for its apertures, presents unique multidisciplinary challenges in the areas of structural dynamics, controls and multi-aperture phasing active optics. In meeting these challenges, emphasis is placed on modularity in spacecraft subsystems and optics as a means of allowing expandability and upgradeability. For the interferometer to function properly, unique methods of coherent wave front sensing are developed and used for error detection in control of the Fast Steering Mirrors (FSMs). The space environment is simulated by floating ARGOS on a frictionless air-bearing that allows it to track fast moving satellites such as the International Space Station (ISS), planets or point stars. A System Identification is performed on ARGOS to determine its dynamic properties and to design optimal controllers for the Attitude Control System (ACS). ACS sensors include an electronic compass with a 2-axis tip-tilt sensor, a viewfinder camera with centroiding algorithm, and a 3-axis rate gyroscope. Nonlinear, quaternionbased control is employed using reaction wheels as the spacecraft's actuators.
\end{abstract}

Keywords: sparse aperture, multiple-aperture optical systems, fizeau interferometer, phased telescope array, attitude control systems, control of optomechanical systems

\section{INTRODUCTION}

The quest for finer angular resolution in astronomy will inevitably lead to larger apertures. Unfortunately, the primary mirror diameter for space telescopes is limited by volume and mass constraints of current launch vehicles as well as the scaling laws of manufacturing costs ${ }^{1}$. Since the cost of monolithic optics increases faster than diameter squared, and mirrors such as the Hubble Space Telescope's are already at the edge of what is financially feasible, efforts are ongoing to break this trend by employing breakthrough technologies such as deployed segmented mirror telescopes, and sparse aperture optics using interferometry. Whereas long baseline stellar Michelson interferometers feed lights from independent collectors to a beam combiner to obtain interfered fringes over a period of time, Fizeau interferometers produce a direct image with full instant $u-v$ coverage. Hence, Fizeau interferometers are suitable for optical imaging of extended objects and rapidly changing targets. In contrast to the long baselines of Michelson interferometers, Fizeau interferometry systems tend to have compact telescope arrays. An optimal imaging configuration designed for sparse arrays was first proposed by Golay ${ }^{2}$. Sparse arrays are promising for applications that do not require extremely high sensitivity (bright source present) and allow for a rather limited field-of-view (FOV) $)^{3,4}$. A notable project in the area of phased telescope array is the Multipurpose Multiple Telescope Testbed (MMTT) ${ }^{5}$ by Air Force Research Laboratory (AFRL). The MMTT consists of four 20$\mathrm{cm}$-aperture telescopes phased together with a 15 -arcmin Field-of-View (FOV). The MMTT employs a complex laser interferometer metrology to sense wavefront

\footnotetext{
* Student Member AIAA, Graduate student, MIT Space Systems Lab, sjchung@mit.edu

† Student Member AIAA, Graduate student, MIT Space Systems Lab, lobosco@mit.edu

* Senior Member AIAA, Associate Professor of Aeronautics and Astronautics, Director of Space Systems Lab, millerd@mit.edu

$\S$ Member AIAA, Research Associate of Aeronautics and Astronautics department, carl@mide.com
} 
error (WFE). The Multi Aperture Imaging Array ${ }^{6}$ built by Lockheed Martin demonstrated phase diversity computation techniques for WFE sensing. This sparse array consists of afocal telescopes arranged in a Y-formation that are combined to a common focus in a Fizeau interferometer configuration. It demonstrated the first results of a broad band multiple telescope imaging array phased over a significant field of view using the extended image projector in the lab. Research into WFE sensing and control has been extensively conducted for the Next Generation Space Telescope (NGST) ${ }^{7}$. The MIT Space Systems Laboratory (SSL) successfully completed the Middeck Active Control Experiment (MACE) ${ }^{10}$, a Space Shuttle flight experiment that flew on STS-67 in March 1995. The primary goal of the MACE project was to investigate approaches for achieving high precision pointing and vibration control of future spacecraft. Built on the heritage of the MACE, a ground-based satellite testbed with an active optical payload system is presented in this paper.

\subsection{Overview of ARGOS}

In order to better understand the technological difficulties involved in designing and building a sparse aperture array, the challenge of building a white light Golay-3 telescope was undertaken. The MIT Adaptive Reconnaissance Golay-3 Optical Satellite (ARGOS) ${ }^{8}$ project exploits wide-angle Fizeau interferometer technology with an emphasis on modularity in the optics and spacecraft subsystems.

Figure 1 highlights the key functional and operational objectives. To demonstrate a complete spacecraft in a 1$\mathrm{g}$ environment, the ARGOS system is mounted on a frictionless air-bearing, and has the ability to track fast orbiting satellites like the International Space Station (ISS) as well as point stars. Modular architecture design emphasizes the use of replicated components and quick connections. The system consists of three identical apertures arranged in a Golay-3 distribution. The light from these telescopes is combined in a center module and
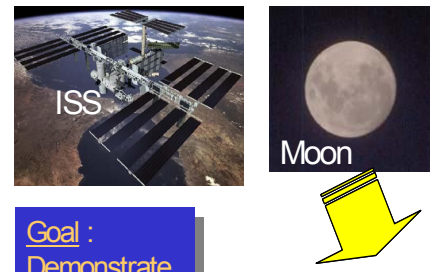

\section{Goal :}

Demonstrate

Practicality of

Modular

Architecture

for Space

Imaging

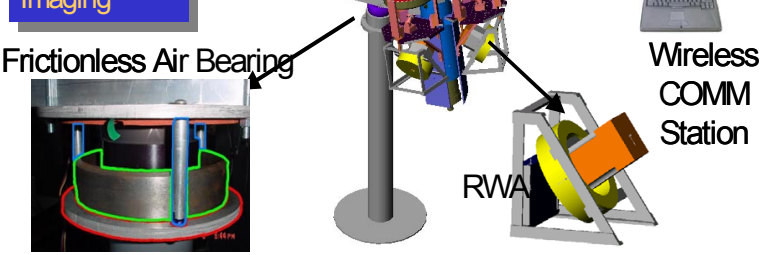

FIG. 1: Overview of ARGOS system

transmitted to a Charge-Coupled Device (CCD). Wavefront sensing techniques are explored to mitigate initial misalignment and to feed back real-time aberrations into the optical control loop. The end result is an image as good as the image received from a monolithic telescope using a single aperture. ARGOS operates autonomously and in a self-contained manner while a wireless ground station downloads images and telemetry information. The primary functional and operational requirements of ARGOS are prescribed in Table 1.

\section{OPTICS SYSTEM DESIGN}

\subsection{Modeling of Beam Combining Errors}

There are three major wavefront errors that need to be controlled at the beam combiner's focus in order to achieve phased beam combining. Those errors are Optical Path Difference (OPD) - piston error, Tilt/Tip error, and lateral pupil mapping error. 
TABLE 1: Key functional and operational requirements

\begin{tabular}{|c|c|}
\hline Key Requirements & \\
\hline \hline Angular Resolution & $0.35 \mathrm{arcsec}$ at visible \\
Operating Wavelength & $400-700 \mathrm{~nm}$ (Visible) \\
Field of View (FOV) & $3 \operatorname{arcmin} * 3 \mathrm{arcmin}$ \\
Field of Regard (FOR) & $120^{\circ}$ \\
Signal to Noise Ratio (SNR) & 100 \\
ACS Pointing Accuracy & $+/-1$ arcmin \\
Image Acquisition Time & 20 images/ hour (max) \\
Autonomous Operation Time & up to 1 continuous hour \\
\hline
\end{tabular}

We can plot the effects of OPD errors using the interferometry equation given by Mennesson ${ }^{9}$.

$$
I \propto\left|\frac{\pi D(1+\cos (r))}{\lambda}\right|^{2}\left|\frac{J_{1}(\pi D \sin r / \lambda)}{\pi D \sin r / \lambda}\right|^{2}\left|\sum_{k=1}^{n} e^{j 2 \pi\left(L_{k} r / \lambda\right) \cos \left(\delta_{k}-\theta\right)} e^{j \phi_{k}}\right|^{\mid}(1)
$$

where $r$ is the off-axis angular direction, $\theta$ is the azimuth angle, $D$ is a subaperture diameter, and $\left(L_{k}, \delta_{k}\right)$ are the polar coordinates of the array configuration. $\phi_{k}$ is the phase shift.

As the piston error increases, two major deviations develop over the envelope of the Point Spread Function (PSF). First, the main envelope shifts in the direction of the piston error. The resultant direction of the envelope shift is the vector sum of piston error directions weighted by the amount of error. Secondly, the peak intensity gets reduced compared to the normal PSF without any piston errors resulting in a reduced Strehl Ratio (SR). The size of the mainlobe also expands showing a degraded angular resolution (See Figure 2). When a piston error is $0.1 \lambda$, the peak intensity is $98 \%$ of the normal intensity. The beam combining piston error tolerance is $0.1 \lambda=55 \mathrm{~nm}$.
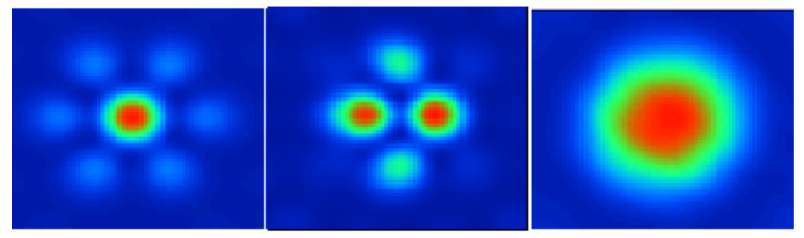

FIG. 2: PSF plot of Golay-3 array with zero OPD, $0.5 \lambda$ OPD, and 1.0 $\lambda$ OPD, from the left to the right.

\subsubsection{Lateral Pupil Mapping Error}

If coherent imaging is to be achieved over any significant field-of-view (FOV), the pupil mapping process must be performed such that the exit pupil is an exact (scaled) replica of the entrance pupil ${ }^{3}$. This constraint is commonly called the golden rule of beam combining.
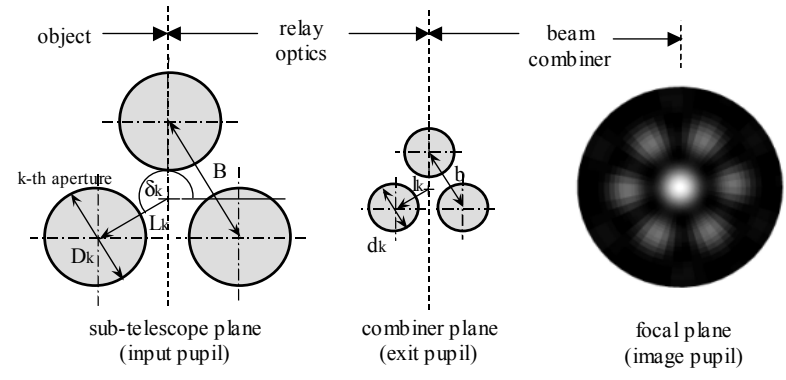

FIG. 3: A golden rule of beam combining, pupil mapping

Depicted in Figure 3, the golden rule of beam combining can be stated as following.

$$
m_{a}=\frac{D}{d} \text { and } m_{t}=\frac{B}{b} \text { Golden Rule: } m_{a}=m_{t}
$$

$D$ is the diameter of a subaperture while $d$ is the com-

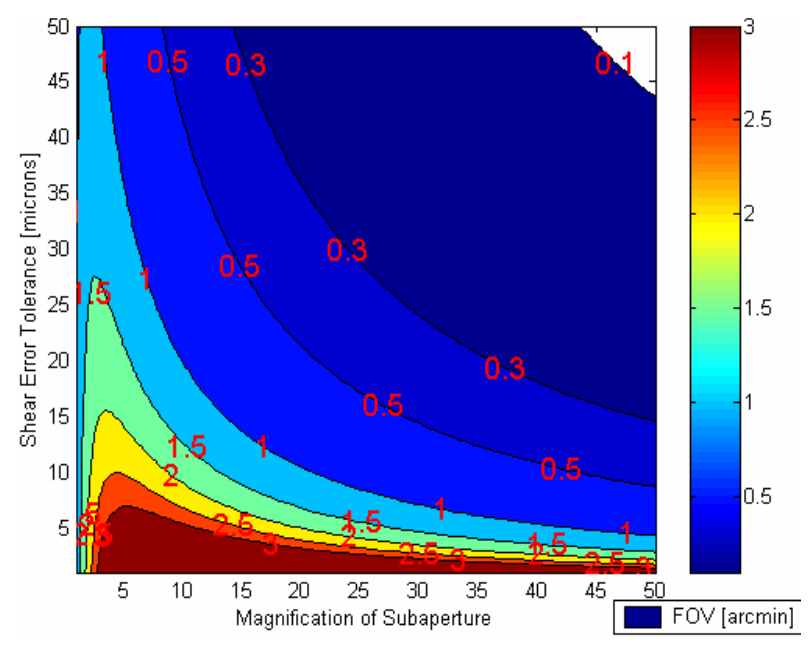

FIG. 4: Tolerable pupil mapping error depending on FOV

pressed exit beam size of a subaperture. $B$ is the baseline length between apertures while $b$ is the distance between compressed beams when they enter the beam combiner. Previously, $12 \mathrm{~mm}$ was suggested for shear error tolerance with an assumption of no magnification error. However, $12 \mathrm{~mm}$ shear error cannot produce the FOV requirement of ARGOS ( 3 arcmin) for any range of magnification error. We can tighten shear error tolerance to meet the FOV requirement or we can relax the FOV requirement by shrinking the region of interest in the whole FOV. Therefore, the pupil mapping process is the primary limiting factor deciding the reasonable FOV of a sparse aperture interferometric array. ARGOS's sub-telescope collimators are designed with a tolerance of 0.0095. However, the ARGOS sub-telescopes have a 
focusing knob which can control the distance between the primary mirror and the secondary mirror, thereby controlling the size of the beam more precisely. It is usually considered that 1/1000 magnification tolerance requirement is too expensive to manufacture. Figure 4 describes how the magnification of a sub-aperture affects the shearing tolerance of a sparse aperture imaging system. This plot implies that sub-aperture magnification can be tuned to maximize allowable shear error (lateral pupil mapping error) thereby reducing control complexity. For a magnification of 10 , we can increase the shear tolerance value sufficiently high at the expense of the reduced FOV.

\subsection{Relay Optics}

The current optical layout of the system is as follows.
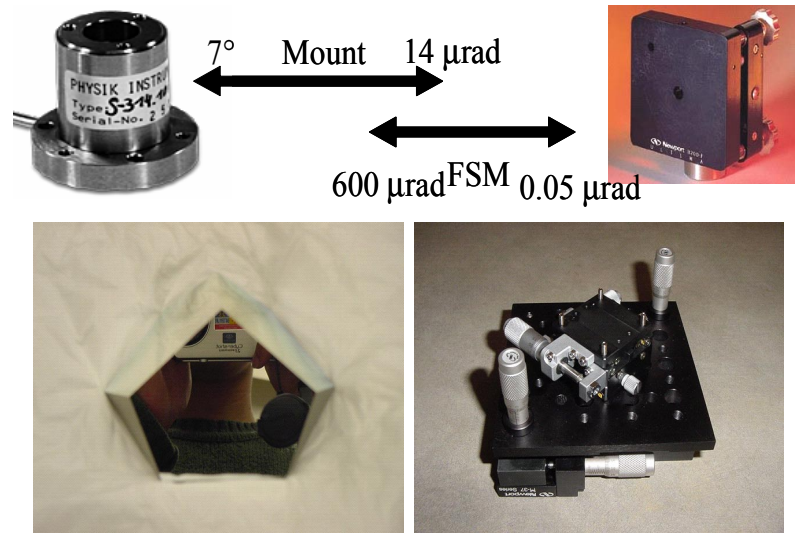

FIG. 5: The range and the resolution of FSM (PI s315.10) and FSM mount (top) and the pyramidal mirror and mount (bottom)

Light shines in through the sub-aperture. The light continues through the telescope until it hits the collimator which is inside the telescopes' baffle. The light then goes through the collimating lens producing a $21 \mathrm{~mm}$ diameter beam. The light exits and hits a reflecting mirror mounted onto a three axis FSM that acts as an ODL as well (See Figure 5). The light is then reflected to the pyramidal mirror that is stationary. The light beam then enters the beam combiner, and is focused onto the CCD.

The FSM has to be able to compensate for any errors in its mounting. Therefore a high precision mount with a range up to 7 degrees was selected for the FSM actuators (See Table 2). The pyramidal mount is composed of two stages. The first stage provides all of the angular adjustments and the second handles $\mathrm{X} \& \mathrm{Y}$ translation in the entrance pupil of the beam combiner. The X-Y translation stage is small enough to fit behind the tip/tilt rotation stage and has the load capacity to hold both the
TABLE 2: Actuator and mount specifications

\begin{tabular}{|c|cccc|}
\hline Model & $\begin{array}{c}\text { Angular } \\
\text { Range }\end{array}$ & $\begin{array}{c}\text { Angular } \\
\text { Resolution }\end{array}$ & $\begin{array}{c}\text { Linear } \\
\text { Range }\end{array}$ & $\begin{array}{c}\text { Linear } \\
\text { Resolution }\end{array}$ \\
\hline \hline FSM & $\pm 600 \mu \mathrm{rad}$ & $\pm 0.05 \mu \mathrm{rad}$ & $12 \mu \mathrm{m}$ & $0.2 \mathrm{~nm}$ \\
& & & & \\
FSM & $\pm 7^{\circ}$ & $\begin{array}{c} \pm 0.0008^{\circ} \\
( \pm 14 \mu \mathrm{rad})\end{array}$ & $1 \mathrm{~cm}$ & $1 \mu \mathrm{m}$ \\
Mount & & & \\
Pyramid & $\pm 4^{\circ}$ & $\begin{array}{c} \pm 2 \mathrm{arcsec} \\
\text { Mirror }\end{array}$ & $13 \mathrm{~mm}$ & $3 \mu \mathrm{m}$ \\
Mount & & $( \pm 9.6 \mu \mathrm{rad})$ & & \\
\hline
\end{tabular}

second stage and the mirror. Any additional $\mathrm{Z}$ directional error can be offset by the FSM mounts.

\subsubsection{Structural Misalignment Tolerancing}

Using the mode of non-sequential ray tracing of ZEMAX, a complete ARGOS optics layout is constructed based on the optical specifications of a subaperture, pyramidal mirror, and the beam combining telescope as shown in Figure 5. We intentionally perturb the sub-telescope or pyramidal mirror to determine allowable structural misalignment, and we compensate the tilt error by changing the tilt angle of the fold mirror attached to the FSM. At 0.01 degree tilt of a subaperture, a pure FSM motion cannot restore the SR (Strehl Ratio) above 0.8 . But the addition of FSM piston motion can restore the SR value to 0.859 . We could achieve a SR of 0.859 (which is above diffraction limited) over 0.01 degree tilt. But due to a magnification factor 10, the FSM compensation exceeded its max range (0.6 $\mathrm{mrad}=0.034$ degree). Since we mounted a FSM onto a precision tip-tip mount which is capable of several arcsecond adjustment (Figure 5 and Table 2), this static error does not limit the FSM performance. However, it is much safer to have a FSM within a range of eliminating a possible maximum alignment error. 0.005 degrees or 15 arcsec for sub-telescope structural misalignment is suggested.

By assuming that all other optical components are perfectly aligned and the FSM can compensate for all the residual tilt errors, the tilt errors for each surface of the pyramidal mirror are calculated. When the tilt error of the pyramidal mirror unit equals the tilt compensation of a FSM, the aberration loss due to the tilt is completely eliminated. Therefore there is no theoretical tilt tolerance for the pyramidal mirror as long as it does not exceeds the maximum compensation range (0.01 degrees).

The beam combiner was tilted along $\mathrm{x}$ and $\mathrm{y}$ axis while leaving other optical components perfectly aligned (Figure 6). This beam combiner misalignment is not correctable by optical actuators like FSMs. However, it 
turns out that we can tolerate up to 0.2 degrees for the beam combiner, which is less stringent than other misalignment tolerances.
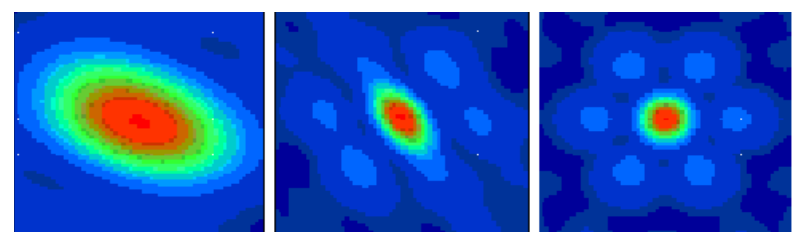

FIG. 6: The PSF plots when the beam combiner has tilt errors. From left to right, (1) X tilt: 0.2 , Y tilt: 0.4 , (2) X: 0.3 Y: 0.3 , (3) X: 0.25 Y: 0.25 [degrees]

\section{ATTITUDE CONTROL SYSTEM (ACS)}

\subsection{ACS Overview}

The final ARGOS structure and the major ACS compo-

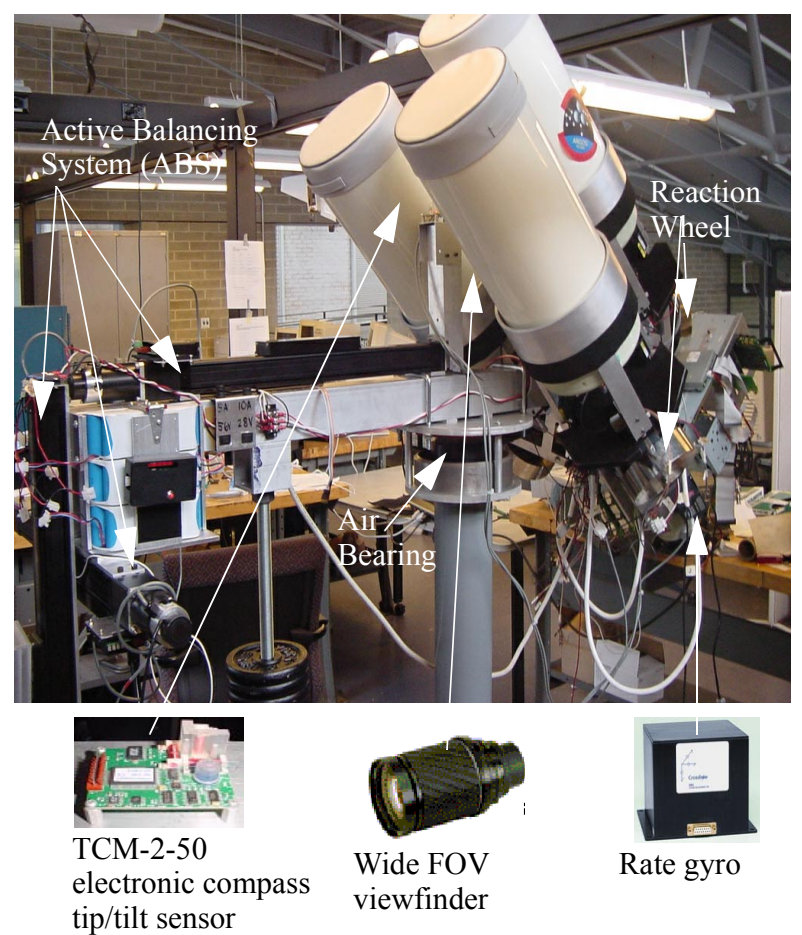

FIG. 7: The final ARGOS system with the three ACS sensors shown in the bottom

nents are depicted in Figure 7. The Field of View of the main CCD is 3 arc-minutes and thus to give us $1 / 2$ arcminute of margin on either side, the ACS subsystem is required to provide a pointing accuracy of $+/-1$ arcminute. The period of operation of the ARGOS system without human intervention must be 60 minutes or greater, meaning that the ACS system will have to either not saturate its actuators, or have some way of desaturating them within this given time span. The system must be able to slew at a rate of at least 1.5 degrees per second, placing a minimum requirement on the capabilities of the actuators to slew the spacecraft.

The sensor suite is composed of three integral elements. First, the TCM-2-50 electronic compass, with a 3-axis magnetometer, a 2-axis tilt sensor, and facilities to provide temperature information. Inclinometers/Electronic compasses measure the relative angles between the inertial coordinate frame and the body fixed frame. That is, they are used to give relative elevation, roll, and azimuth information between these two coordinate systems. The Inclinometer/Electronic compass is an essential component of the coarse pointing sensor suite. Second, there is an intermediary sensor, which takes the form of a scope. It is the intermediate wide FOV CCD that provides sufficient overlap (greater than 4) between the TCM (Figure 8) and the main imager. Thirdly, there is a 3-axis rate gyroscope for angular rate measurement.

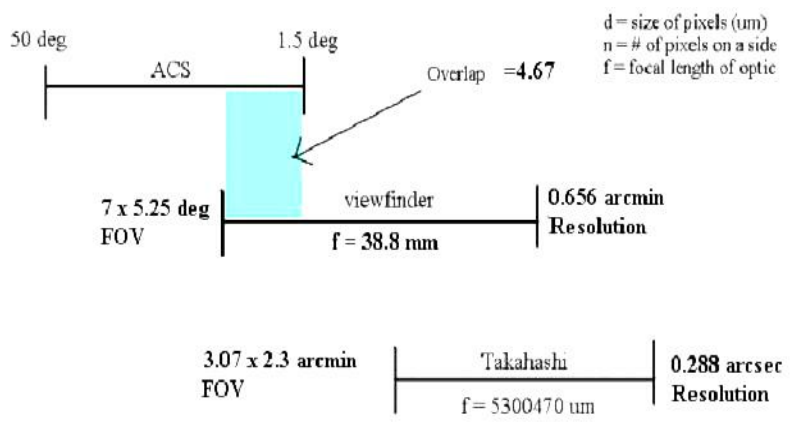

FIG. 8: Multi-staged ACS sensors

\subsection{Active Balancing System (ABS)}

Due to the nature of the air bearing system chosen to simulate the space-based operation of ARGOS, the center of gravity and the center of rotation of the body will not necessarily be at the same position. The offset between their positions will impart a gravitational torque on the spacecraft, which will need to be overcome by the reaction wheels, both increasing their torque requirements as well as significantly increasing the angular momentum storage required by the reaction wheels.

The torque causes the wheels to increase in speed according to the equation:

$$
\Delta \dot{\theta}_{\text {wheel }}=\frac{m_{s / c} g T_{\text {desat }}}{I_{\text {wheel }}} \delta
$$




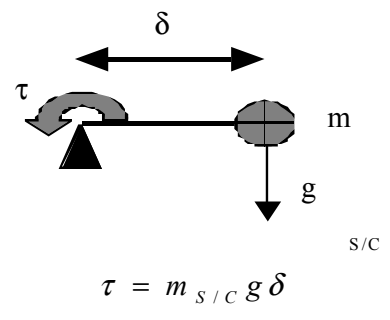

FIG. 9: Schematic of center of rotation, center of gravity offset, and gravitational torque.

$\Delta \dot{\theta}$, the change in wheel speed before desaturation is required, is set by a combination of the limitations on the reaction wheel motor and the degree to which the wheels are balanced. $T_{\text {desat }}$ is the time between desaturations and is set as a requirement for the overall ARGOS system. In designing the reaction wheels, it was intended to have the primary drivers be similar to those encountered in a space environment. Therefore, $I_{\text {wheel }}$ should not be made much larger than would otherwise be required to control the dynamics of the spacecraft without having to overcome gravitational torques greatly exceeding those encountered in a space environment. In order to meet these constraints, the offset between the center of gravity and the center of rotation ( $\delta)$ must be kept to on the order of $1 \mu \mathrm{m}$.

Statically balancing by designing for zero offset and using small ballast weights to make corrections may not be sufficient to achieve this. As such, an active balancing system, which can cause the overall spacecraft center of mass to shift, has been devised. At the start of operation, an automatic balancing routine is run. The

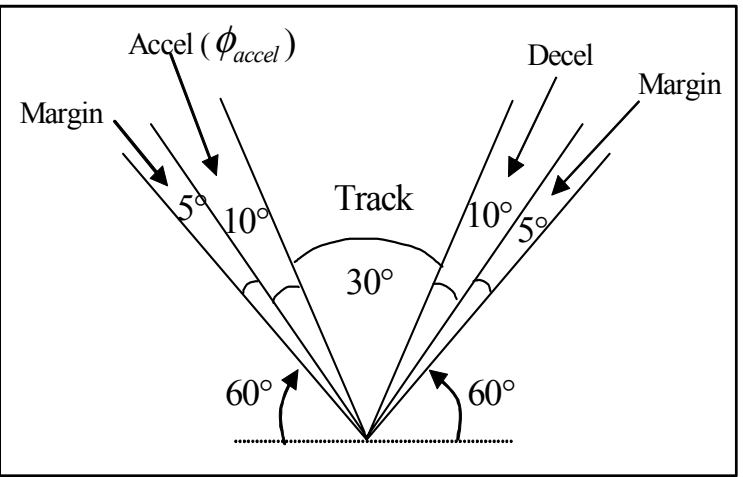

FIG. 10: ISS tracking operation

ABS corrects for small center of mass offsets that would hurt the closed-loop performance. The system can also be used to remove momentum from the wheels by intentionally causing an offset in the appropriate direction. This does not work about the vertical axis since one cannot put a gravitational torque about it. There are also less disturbance torques about this axis, meaning that momentum build-up about this axis will be minimal. The selected active balancing system design will use three linear motion slides based upon the custom developed lead screw and fixed servo system selected from the trades analysis. The slides are positioned on the end of the swing arm away from the telescope, since they would not be included on an actual spacecraft. Each of the slides will be mounted perpendicular to the other two, such that there are three independent axes of control of the position of the center of gravity (See Figure 7). 


\subsection{Control Hardware and Avionics}

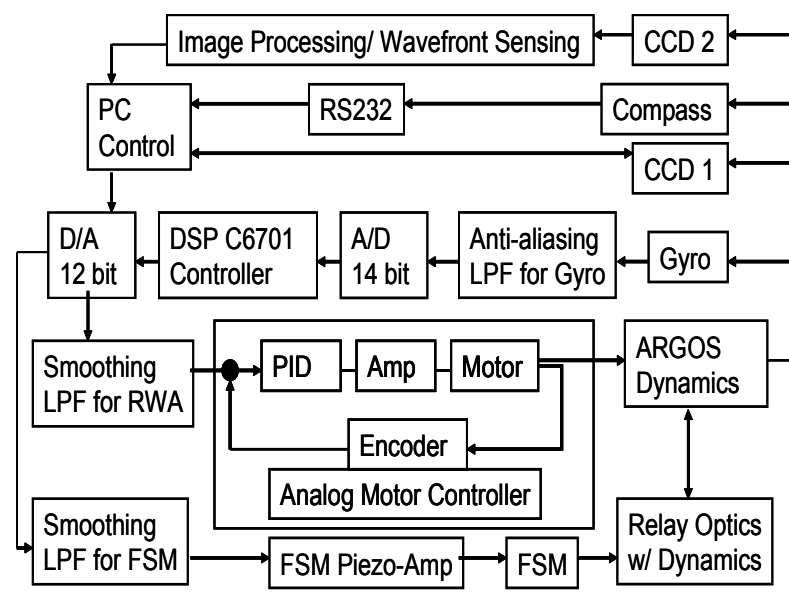

FIG. 11: Control avionics schematic diagram

Figure 11 contains the ARGOS avionics hardware sche-

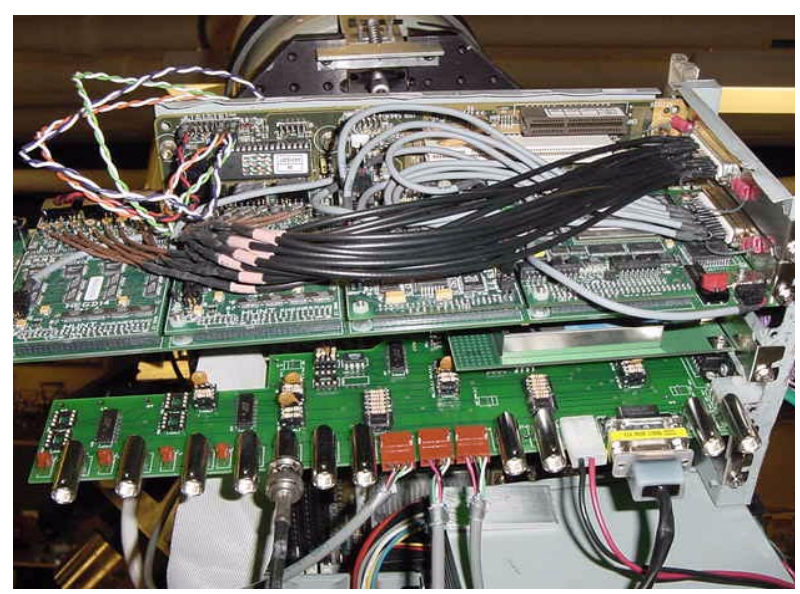

FIG. 12: DSP and Heron D/A, A/D modules (top) and ACS filter board (bottom)

matic. The HEPC8 digital signal processing module houses a TI C6701 DSP, two 12bit 8-channal D/A (3 signals for each FSM and 3 RWA) and one 14bit 8-channel A/D as shown in Figure 12.

Each reaction wheel speed command from the DSP is sent through smoothing filters with a cut-off frequency of $280 \mathrm{~Hz}$. The internal analog motor controller employs a PID logic using the motor encoder sensor outputs. A set of three piezoelectric stacks for a single aperture FSM (a total of 9 piezo linear stacks) is driven by the D/ A signals, which are filtered by a $303 \mathrm{~Hz}$ cut-off frequency low pass filter and then amplified by the compact voltage amplifier (PI E0669.OE) located in the right side of ARGOS. The rate gyroscope measures the rotational rates of ARGOS and feedbacks the voltages to the A/D with a resolution of $50 \mathrm{mV} /($ degrees $/ \mathrm{sec})$. An anti-aliasing low pass filter with a $48 \mathrm{~Hz}$ cut-off frequency was implemented to reduce the high-frequency noise sent to the $\mathrm{A} / \mathrm{D}$ channels. The electronic compass is directly connected to the PC motherboard using a standard RS232 serial port. The PC-DSP FIFO designated for direct communication between the host PC memory and the DSP memory, sends the image processing data ${ }^{8}$ such as the centroids of the target from the intermediate $\mathrm{CCD}$, and the compass readings to the DSP controller in real-time.

To meet the requirement of simultaneous automatic control of various subsystems, the DSP modules, the ABS controller and the wireless card for the communication with a remote lap-top control ground station, all reside in the PCI slots of the Athlon $1.4 \mathrm{GHz}$ PC system with 1024 MB RAM. The GUI PC control application sends the reference slew speed and pointing (azimuth and elevation) for a specific target to the DSP while performing real-time image processing from both the intermediate ACS CCD and the main imager (spot-based wavefront sensing ${ }^{8}$ ).

\section{ACS CONTROL DESIGN AND RESULTS}

\subsection{System Identification}

The objective of a System Identification is to determine the dynamic properties of ARGOS and correctly design an appropriate control system to capture all present issues that would deteriorate the performance. The $3 \times 3$ transfer function matrix between the three D/A signals from the DSP and the three gyro outputs to the A/D port for the RWA control (See Figure 11) is obtained. In this case, the System Identification will give us a transfer function with the ACS filter board reference command as the input, the smoothing low pass filter, motor controller, reaction wheels, structure, rate gyros, and antialiasing low pass filter as the plant, and the filtered gyro signal from the ACS filter board as the output.

The transfer function of the plant is then found by dividing the cross spectral density of the input and output by the power spectral density of the input. Then, the measurement-based system identification algorithm called Integrated Frequency domain Observability Range Space Extraction and Least Square parameter estimation algorithm (IFORSELS) ${ }^{10,11}$, is performed to derive a state-space representation of the system dynamics. IFORSELS algorithm integrates the Frequency domain Observability Range Space Extraction (FORSE) identi- 

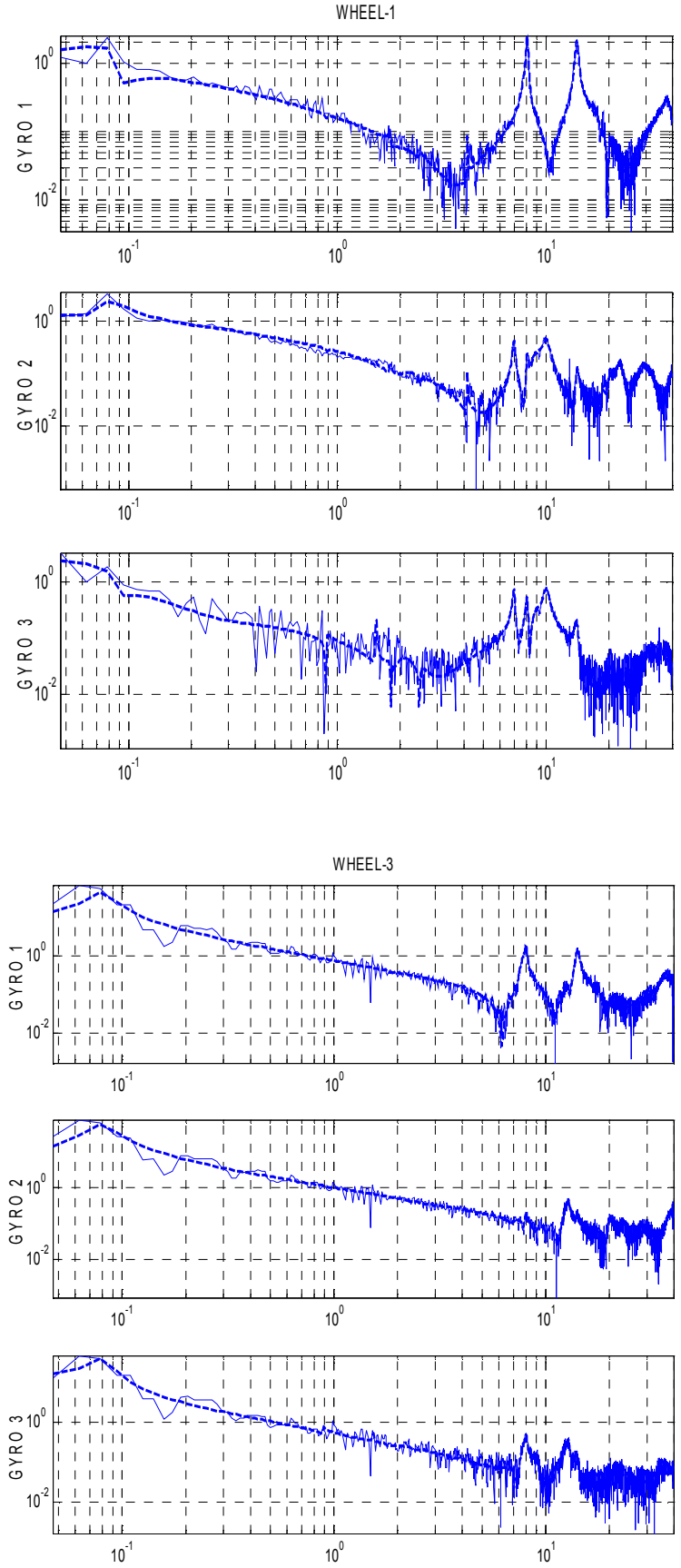

FIG. 13: System Identification of ARGOS (R/W 1 and 3) to Gyro 1,2,3 outputs (frequency in $\mathrm{Hz}$ ). Due to the symmetry, R/W 2 is equivalent to R/W 1 .

fication algorithm, the Balanced Realization (BR) model reduction algorithm, and the logarithmic and additive Least Squares (LLS) modal parameter estimation algorithms for low order highly accurate model identification.
The FORSE algorithm is a slight variation of the subspace identification algorithm derived by De Moor, et $\mathrm{al}^{12}$ and its objective is to minimize the following cost to obtain the system matrices, $A, B, C, D$,

$$
J=\sum_{k=1}^{K}\left\|\hat{G}\left(\omega_{k}\right)-\left(C\left(e^{j \omega_{k} \Delta t} I-A\right)^{-1} B+D\right)\right\|_{2}^{2}
$$

where $\hat{G}\left(\omega_{k}\right)$ is the frequency response samples from experiments.

The BR model reduction ${ }^{13}$ algorithm transforms a state space model to a balanced coordinate and reduces the model by deleting the states associated with the smallest balanced singular values. The LLS estimation algorithms improve the fitting of reduced models to experimental data by updating state space parameters in modal coordinates. IFORSELS was developed at the MIT SSL for the MACE experiment and is now licensed by Midé Technology Corporation (Dynamod Matlab toolbox).

From the experimental transfer function measurements, a $0.01 \mathrm{~Hz}$ to $40 \mathrm{~Hz}$ chirp signal generated the sampled transfer functions in Figure 13 (solid line). Using the FORSE algorithm, an 80th order state-space model was selected to approximate the experimental data and then BR and LLS algorithms were used to get a 60th order IFORSELS model (shown by dotted lines in Figure 13). The low-frequency peaks below $0.1 \mathrm{~Hz}$ is the pendulum mode of ARGOS while the first lightly-damped structural mode occurs at $8-9 \mathrm{~Hz}$.

\subsection{Nonlinear Controller Design}

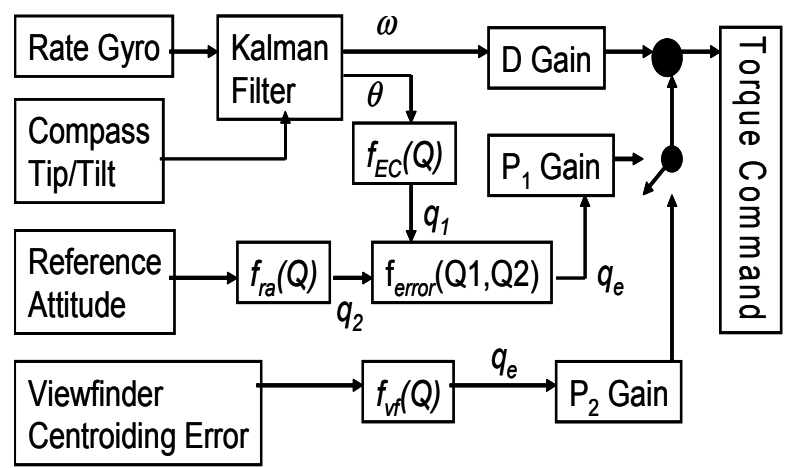

FIG. 14: ARGOS ACS general control design

A summary of the control design of the ACS subsystem is presented as shown in Figure 14. Through the control method the attitude of the satellite can be deciphered and controlled. Initially the ARGOS satellite is at some 
arbitrary position, then the Science Operations and Communications subsystem provides reference information as to the position and behavior of the satellite by providing the ACS subsystem with the International Space Station's azimuth, elevation, range, azimuth rate and elevation rate. The ACS subsystem then converts this information into the body frame of ARGOS and then converts the input information into quaternion form $\left(f_{r a}(Q)\right.$ in Figure 14). At the same time, two of the sensors being used, the rate gyroscope and electronic compass, are providing information as to the actual attitude of the satellite, this information is similarly ran through a function that transforms it into the body frame if necessary and then converts it to quaternion form $\left(f_{E C}(Q)\right.$ in Figure 14). With this information the error in ARGOS's actual attitude can be computed and based on the desired and actual attitude information, how much the ARGOS system would need to rotate from its current body frame to the desired body frame would be known $\left(f_{\text {error }}(Q 1, Q 2)\right.$ in Figure 14). The error quaternion is defined by:

$$
\left[\begin{array}{l}
q_{1} \\
q_{2} \\
q_{3} \\
q_{4}
\end{array}\right]_{e}=\left[\begin{array}{cccc}
q_{d 4} & q_{d 3} & -q_{d 2} & q_{d 1} \\
-q_{d 3} & q_{d 4} & q_{d 1} & q_{d 2} \\
q_{d 2} & -q_{d 1} & q_{d 4} & q_{d 3} \\
-q_{d 1} & -q_{d 2} & -q_{d 3} & q_{d 4}
\end{array}\right]\left[\begin{array}{c}
-q_{1} \\
-q_{2} \\
-q_{3} \\
q_{4}
\end{array}\right]_{\text {compass }}
$$

where $q_{d}$ is the desired quaternion.

The error quaternion is passed through an attitude controller which exhibits proportional, integral and derivative control; it is based on a "PD like" non-linear control design. The output from the attitude controller is wheel speed and this is fed through an actuator system that outputs torque. This torque is then fed through the Attitude Control plant (the physical system) after which the body rate and body rotation of ARGOS would be known.

Once the ISS is within the ACS viewfinder's field-ofview, the ACS system moves into the second mode. The centroding algorithm relays through the viewfinder the position of the ISS in $(\mathrm{x}, \mathrm{y})$ Cartesian coordinates. This viewfinder input is again transformed into ARGOS's body frame and converted into quaternion form $\left(f_{v f}(Q)\right.$ in Figure 14). Thus in the second mode we only have rate gyro and viewfinder input. In both modes, a Kalman filter ${ }^{14,15,16}$ is used to provide the best representation of the actual system attitude and rate by combining the input from the different sensors in the optimal way. For example, inclinometer input is best at low frequencies while rate gyro input is best at high frequencies, thus a mixing filter such as the Kalman filter becomes necessary to output the best combination of the two inputs.

\section{CONCLUSION AND FUTURE WORK}

The ARGOS is successfully designed and integrated into the full structure and is ready to operate. The controller design in this paper is focused on the attitude control system incorporating the coarse optical sensor. The fine active optics controller utilizing model-based control and neural network is under development. A preliminary assessment shows that the beam combining problem is the most challenging aspect of sparse optical arrays. The need for optical control is paramount due to tight beam combining error tolerances. The wavefront sensing/control requirements appear to be a major technology and cost driver. The ARGOS testbed uniquely addresses the real world problems such as the vibrational coupling between a spacecraft structure and the wavefront errors propagating through the whole system. The LQG-type controller for the rate-damping keeping by using the system identification results would be explored soon, however, the nonlinearity in quaternion representations make it difficult to implement a pure linear controller for large angle rotations.

\section{ACKNOWLEDGEMENTS}

This research was supported by the NRO Director's Innovation Initiative (DII) and Mide Technology Corporation. Authors would like to thank Alice K. Liu at NASA Goddard Space Flight Center for her initial contribution to the ARGOS attitude control system.

\section{References}

1. Meinel, A.B. , "Cost Scaling Laws Applicable to Very Large Telescopes", SPIE Proceedings Vol. 172, 1979.

2. Golay, M., "Point Arrays Having Compact Nonredundant Autocorrelations", J.Opt Soc. Am., vol. 61, pp 272, 1971.

3. Harvey, J.E., Silverglate, P.R., and Wissinger, A.B., "Optical Performance of Synthetic Aperture Telescope Configurations", Southwest Conference in Optics, pp 110-118, SPIE vol. 540, 1985.

4. Faucherre, M., Merkle, F., and Vakili, F., "Beam Combination in Aperture Synthesis from Space: Field of View Limitations and (u,v) Plane Coverage Optimization", New Technologies for Astronomy, pp 138-145, SPIE vol. 1130, 1989.

5. De Hainaut, C. R., et al, "Wide Field Performance of a Phased Array Telescope", Optical Engineering, vol. 34 No.3, March 1995.

6. Zarifis, V., et al, " The Multi Aperture Imaging Array", ASP Conf. Series 194, Working on the Fringe: Optical and IR Interferometry from Ground and Space, 
Unwin and Stachnik, eds, pp. 278.

${ }^{7}$ Redding, D. C., et al, "Wavefront sensing and control for a Next-Generation Space Telescope", Proc. SPIE Vol. 3356, p. 758-772, 1998.

${ }^{8}$ Chung, S.-J., "Design, Implementation and Control of a Sparse Aperture Imaging Satellite", Master of Science Thesis, Department of Aeronautics and Astronautics, MIT, 2002.

9 Mennesson, B., and Mariotti, J.M., " Array Configurations for a Space Infrared Nulling Interferometer Dedicated to the Search for Earthlike Extrasolar Planets", ICARUS 128, pp 202-212, 1997.

${ }^{10}$ Miller, D. W., et al, "The Middeck Active Control Experiment(MACE): Summary Report," Space Engineering Center, MIT, SERC\#7-96 .

${ }^{11}$ Liu, K., Jacques, R. N., and Miller, D. W.,"Frequency Domain Structural System Identification by Observability Range Space Extraction," ASME Journal of Dynamic Systems, Measurement, and Control, June 1996.
12 De Moor, B., et al, "A Geometrical Approach for the Identification of State Space Models with Singular Value Decomposition," Proceedings, IFAC Symposium on Identification and System Parameter Estimation, Aug. 1998, Beijing, China.

${ }^{13}$ Zhou, K., Doyle, J. C., and Glover, K., Robust and Optimal Control, Prentice Hall, Upper Saddle River, NJ, 1996.

${ }^{14}$ Franklin, G. F., Powell, J. D., and Workman, M., Digital Control of Dynamic Systems, 3rd ed., Addison Wesley, Menlo Park, CA, 1997.

${ }^{15}$ Brown, R.G. and Hwang, P.Y.C., Introduction to Random Signals and Applied Kalman Filtering, 3rd ed., John Wiley \& Sons, 1997.

${ }^{16}$ Marins, J. L., et al., "An Extended Kalman Filter for Quaternion-Based Orientation Estimation Using MARG Sensros", Proc. 2001 IEEE/RSJ International Conference on Intelligent Robots and Systems, Maui, HI, 2001 . 